\title{
MÉTODOS DE APREÇAMENTO DE OPÇÕES AMERICANAS E DETERMINAÇÃO DA CURVA DE GATILHO ATRAVÉS DA SIMULAÇÃO DE MONTE CARLO
}

\author{
Javier Gutiérrez Castro* \\ Tara Keshar Nanda Baidya \\ Departamento de Engenharia Industrial (DEI) \\ Pontifícia Univ. Católica do Rio de Janeiro (PUC-Rio) \\ Rio de Janeiro - RJ \\ javiergc@aluno.puc-rio.br; baidya@puc-rio.br \\ Fernando Antonio Lucena Aiube \\ DEI / PUC-Rio e Petrobras \\ Rio de Janeiro - RJ \\ aiube@puc-rio.br \\ * Corresponding author / autor para quem as correspondências devem ser encaminhadas \\ Recebido em 07/2007; aceito em 06/2008 \\ Received July 2007; accepted June 2008
}

\section{Resumo}

Nos últimos anos a simulação de Monte Carlo tem se constituído numa das principais ferramentas que analistas acadêmicos utilizam com fins de apreçamento de derivativos. A principal motivação é a grande flexibilidade que apresenta para simular diversos tipos de opções e preços do ativo subjacente. Neste trabalho são analisados três algoritmos para o cálculo de opções de venda americanas: Ibáñez \& Zapatero (2004), Ibáñez \& Zapatero modificado (aqui proposto) e o método LSM (Least Square Monte Carlo) de Longstaff \& Schwartz (2001). O método proposto oferece resultados tão bons como o LSM com a vantagem adicional de permitir o cálculo da curva de gatilho (threshold curve). O método LSM é de rápido processamento computacional no cálculo do preço da opção, mas não gera em primeira instância uma curva de gatilho, a qual, para alguns casos (como na análise de investimentos) é muito útil na definição do instante ótimo de investir.

Palavras-chave: derivativos financeiros; opções de venda americanas; simulação de Monte Carlo.

\begin{abstract}
Monte Carlo Simulation has become one of the most important tools used in pricing Financial Derivatives. The main reason in that it gives greater flexibility in simulating several kinds of underlying assets and derivatives. In this article, we analyze three algorithms to calculate the value of American options, which is one particular type of financial derivatives. The first two are: that of Ibáñez \& Zapatero (2004) and that of Longstaff \& Schwartz (2001). The third algorithm is the one we have developed by modifying Ibáñez \& Zapatero. Our method gives results which are as good as those of Longstaff \& Schwartz, with the additional advantage that it calculates the threshold curve. The threshold curve is very important for defining the optimal timing of investments. The method of Longstaff \& Schwartz has a fast computational process to calculate option price, but it doesn't produce a threshold curve.
\end{abstract}

Keywords: financial derivatives; American put options; Monte Carlo simulation. 


\section{Introdução}

A Simulação de Monte Carlo é uma metodologia muito difundida para o apreçamento de derivativos financeiros e cálculo do valor de opções reais em projetos de investimento. Em ambas as aplicações é importante que o algoritmo seja computacionalmente eficiente, e que, quando empregado em opções reais, seja capaz de estimar o instante ótimo de investir. A determinação dos instantes ótimos de investimento ao longo de todo o período de maturação da opção define o que na literatura é conhecido como "curva de gatilho" (threshold curve).

Por exemplo, é possível identificar o período adequado para realizar um investimento de valor K. Este ocorre quando o valor do projeto chega a um nível igual ou superior àquele definido na curva. Outro exemplo, é o caso em que existe a possibilidade de abandonar o projeto (obtendo um valor de recuperação $\mathrm{K}$ ), sendo que a opção de abandono deverá ser exercida assim que o valor do projeto atinja um nível igual ou inferior àquele definido na curva. Estes são alguns exemplos da utilidade prática da curva de gatilho.

Entre os diversos trabalhos desenvolvidos em simulação de Monte Carlo para opções americanas, a grande maioria deles tenta aproximar o preço da opção através da programação dinâmica, isto é, utilizando procedimentos recursivos de cálculo (de trás para frente) a partir de simulações dos valores do ativo subjacente ao longo do tempo até a data de expiração da opção. Nesse contexto, as metodologias elaboradas por Boyle et al. (1997) e Broadie \& Glasserman (1997) constituem referências para as pesquisas que posteriormente se desenvolveram no aprimoramento do cálculo do preço de uma opção americana por meio da simulação de Monte Carlo.

Seguindo esta linha, um método amplamente difundido pela sua facilidade de aplicação (mas computacionalmente intenso), é o desenvolvido por Grant et al. (1997). Embora o algoritmo de Grant et al. (1997) consiga gerar resultados razoavelmente aceitáveis (ver testes feitos em Frota (2003)), este se torna bastante intenso computacionalmente e passível de gerar erros de precisão no cálculo recursivo da curva de gatilho, porque não há uma regra explícita que garanta uma boa convergência para os instantes ótimos de investimento.

Outro método (ultimamente muito em voga) que determina o preço de uma opção americana, é o desenvolvido por Longstaff \& Schwartz (2001). A metodologia chamada de LSM (Least Square Monte Carlo) utiliza também o critério da programação dinâmica, mas o procedimento dispensa a determinação prévia da curva de gatilho.

Uma metodologia alternativa foi desenvolvida por Ibáñez \& Zapatero (2004). Como em Grant et al. (1997), primeiramente se determina a curva de gatilho, mas utilizam um procedimento diferente para obter a convergência até os pontos que constituem tal curva.

Neste artigo se analisa com especial ênfase o algoritmo de Ibáñez \& Zapatero (2004). Por outro lado, foram feitas modificações que aprimoram a construção da curva de gatilho. Isto permitiu melhorar a precisão dos resultados usando os testes originais propostos pelos autores. Particularmente, o enfoque deste trabalho está na avaliação de opções de venda americanas com uma variável estocástica. São comparadas três metodologias: Ibáñez \& Zapatero (2004), Ibáñez \& Zapatero modificado (aqui proposto) e o algoritmo de Longstaff \& Schwartz (2001).

$\mathrm{O}$ artigo foi organizado da seguinte forma: a seção 2 faz uma breve revisão teórica sobre opções americanas, a seção 3 descreve o algoritmo de Ibáñez \& Zapatero (2004) explicando os conceitos básicos de curva de gatilho e o método de aproximações sucessivas de Newton; 
a seção 4 aplica o algoritmo descrito na seção anterior, com as modificações propostas apresentando testes numéricos; a seção 5 realiza idênticos testes aplicando o algoritmo LSM; a seção 6 compara os resultados obtidos com as diferentes metodologias; e a seção sete apresenta as conclusões e considerações finais.

\section{Considerações Teóricas}

Em opções financeiras ou reais existe um intervalo de tempo para o exercício da opção, que vai de $\mathrm{t}_{0}=0$, até $\mathrm{t}_{\mathrm{N}}=\mathrm{T}$ (tempo de maturidade). $\mathrm{O}$ prazo até a maturidade ou vencimento é discretizado em $\mathrm{N}$ intervalos, sendo que a opção pode ser exercida em qualquer um destes intervalos. Quando se emprega a simulação para calcular o preço de uma opção americana, o tratamento dado assemelha-se a uma opção bermuda, caracterizada pela possibilidade de ter mais de uma possível data de exercício até sua maturidade. Quanto mais intervalos discretos forem considerados no período $\left[\mathrm{t}_{0} ; \mathrm{T}\right]$, melhor será o modelo que descreve o comportamento real de uma opção americana (que se exerce em tempo contínuo e não discreto).

O método de Grant et al. (1997) determina o preço crítico de exercício $\left(\mathrm{S}_{\mathrm{t}_{\mathrm{i}}}{ }^{*}\right)$ para cada instante de tempo $t_{i}$, onde i $€\{0,1,2, \ldots, N-1\}$. O preço crítico de exercício é o preço da ação ou ativo subjacente (S) que faz com que o valor de exercer a opção em um determinado tempo $t_{i}$ seja igual ao valor esperado de manter viva a opção para ser exercida em uma data posterior. Uma vez determinados os preços críticos em todos os intervalos de tempo, calcula-se de maneira recursiva o preço da opção, empregando um simples processo de simulação que é descrito na seguinte seção. O conjunto formado pelos diferentes preços críticos de exercício, a cada instante de tempo $\mathrm{t}_{\mathrm{i}}$, constitui a curva de gatilho ou fronteira de exercício ótima.

No método LSM de Longstaff \& Schwartz (2001) são realizadas simulações do preço do ativo subjacente ao longo do tempo a partir de um valor inicial, e, por meio de uma análise recursiva a cada instante de tempo $t_{n}(n \in\{\mathrm{N}-1, \mathrm{~N}-2, \ldots, 1\})$ anterior à data de maturação da opção americana, compara-se o valor intrínseco (valor da opção se exercer no instante $t_{n}$ ) com o valor de continuação (valor da opção se esperar para exercer num tempo posterior a $\mathrm{t}_{\mathrm{n}}$ ). $\mathrm{O}$ valor de continuação é estimado através do valor esperado proveniente de uma regressão de mínimos quadrados entre os preços do ativo $\mathrm{S}$ que estão no dinheiro (in the money), ou seja aqueles em que a opção tem valor e pode ser exercida em $t_{n}$, versus 0 valor da opção em um instante $t_{n+1}$, descontado pela taxa livre de risco ao tempo $t_{n}$. Estas comparações se realizam a cada tempo $t_{n}$ até $t_{1}$. Assim, se existem $\mathrm{N}$ datas de exercício, serão necessárias realizar $\mathrm{N}-1$ regressões (em tempos: $\mathrm{t}_{\mathrm{N}-1}, \mathrm{t}_{\mathrm{N}-2}, \ldots, \mathrm{t}_{1}$ ). Discute-se muito sobre a forma dos polinômios de regressão e o grau que eles devem ter, mas foi verificado experimentalmente que polinômios lineares simples de baixo grau têm bom desempenho na estimação do valor de continuação. Por outro lado, cabe ressaltar que, de acordo com os experimentos numéricos feitos em Frota (2003) e Nascimento (2005), foi demonstrado que a metodologia LSM oferece resultados mais exatos do que o método de Grant et al. (1997) consumindo um tempo computacional muito menor.

No que diz respeito à metodologia desenvolvida por Ibáñez \& Zapatero (2004) eles determinam previamente a curva de gatilho para calcular o preço da opção americana. A novidade que estes autores trazem, em relação a Grant et al. (1997), é a utilização do método de aproximações sucessivas de Newton para atingir os preços críticos de exercício, o qual se mostra ser muito eficiente. Outra vantagem deste algoritmo é a possibilidade de estender o cálculo do preço da opção para casos com múltiplas variáveis estocásticas.

Pesquisa Operacional, v.28, n.3, p.473-490, Setembro a Dezembro de 2008 


\section{O Algoritmo de Ibáñez \& Zapatero (2004)}

Nesta seção explicamos o procedimento desenvolvido por Ibáñez \& Zapatero (2004). Descreve-se o algoritmo para o caso de uma opção de venda americana com única variável estocástica (o preço do ativo subjacente). Os autores apresentam o algoritmo de uma maneira generalizada, considerando a possibilidade de existir mais de uma variável estocástica, sendo que o preço da opção americana (de compra ou venda) pode ser descrito em função destas variáveis.

\subsection{Definição das variáveis e parâmetros}

Denote-se por $\mathrm{P}_{\mathrm{t}}\left(\mathrm{S}_{\mathrm{t}}, \mathrm{K}\right)$ o preço da opção e $\mathrm{I}\left(\mathrm{S}_{\mathrm{t}}, \mathrm{K}\right)=\mathrm{K}-\mathrm{S}_{\mathrm{t}}$ o valor intrínseco, onde $\mathrm{S}_{\mathrm{t}}$ é o valor do ativo subjacente, que é uma variável estocástica, e K é o preço de exercício.

$\mathrm{O}$ algoritmo determina primeiramente a curva de gatilho ou fronteira de exercício ótima que é formada pelo conjunto de pontos $\mathrm{S}_{\mathrm{t}}{ }^{*}$ nos quais o valor de manter viva a opção (esperar) é igual ao valor intrínseco (exercer), isto é, $\mathrm{P}_{\mathrm{t}}\left(\mathrm{S}_{\mathrm{t}}{ }^{*}, \mathrm{~K}\right)=\mathrm{I}\left(\mathrm{S}_{\mathrm{t}}{ }^{*}, \mathrm{~K}\right)$, sendo que no momento do exercício $\mathrm{I}\left(\mathrm{S}_{\mathrm{t}}, \mathrm{K}\right)=\mathrm{K}-\mathrm{S}_{\mathrm{t}}$ no caso de uma opção de venda.

Dado um instante inicial $t_{0}$, e um instante $\mathrm{T}$ (maturidade da opção ou prazo máximo de exercício), pode-se subdividir o horizonte de tempo $\mathrm{T}-\mathrm{t}_{0} \mathrm{em} \mathrm{N}$ intervalos, com datas de exercício discretas em $\left\{t_{1}, t_{2}, \ldots, t_{N}=T\right\}$. Em algum instante de tempo $t_{n}$, assume-se que $o$ exercício da opção é ótimo se $\mathrm{S}_{\mathrm{t}_{\mathrm{n}}} \leq \mathrm{S}_{\mathrm{t}_{\mathrm{n}}}{ }^{*}$.

Seja $\mathrm{r}$ a taxa livre de risco de curto prazo, e $\mathrm{Q}$ a medida de probabilidade martingale. Logo, em alguma data $\mathrm{t}_{\mathrm{n}}(\mathrm{n} \epsilon\{\mathrm{N}-1, \mathrm{~N}-2, \ldots, 1\})$, o preço da opção é calculado por:

$$
\mathrm{P}_{\mathrm{t}_{\mathrm{n}}}\left(\mathrm{S}_{\mathrm{t}_{\mathrm{n}}}, \mathrm{K}\right)=\mathrm{E}_{\mathrm{t}_{\mathrm{n}}}^{\mathrm{Q}}\left[\mathrm{e}^{-\mathrm{r} \int_{t=\mathrm{t}_{\mathrm{n}}^{*}}^{*^{*} \mathrm{dt}}} \mathrm{I}\left(\mathrm{S}_{\tau^{*}}, \mathrm{~K}\right)\right]
$$

Onde $\tau^{*} \in\left\{\mathrm{t}_{\mathrm{n}+1}, \mathrm{t}_{\mathrm{n}+2}, \ldots, \mathrm{T}\right\}$ é o chamado tempo ótimo de parada, definido como o primeiro $\mathrm{t}_{\mathrm{n}+\mathrm{i}}$ no qual $\mathrm{S}_{\mathrm{t}_{\mathrm{n}+\mathrm{i}}} \leq \mathrm{S}_{\mathrm{t}_{\mathrm{n}+\mathrm{i}}}{ }^{*}$; noutro caso $\tau^{*}=\infty$. Em outras palavras, é o primeiro instante em que o preço do ativo "S" fica abaixo da curva de gatilho.

Assumindo que $\mathrm{S}_{\mathrm{t}}$ segue um Movimento Geométrico Browniano (MGB), sob a medida martingale, escreve-se: $\mathrm{dS}_{\mathrm{t}} / \mathrm{S}_{\mathrm{t}}=\alpha \mathrm{dt}+\sigma \mathrm{dZ}$, onde $\alpha=\mathrm{r}$-q é o drift ou tendência neutra ao risco, $\mathrm{q}$ taxa de dividendos, $\sigma$ é a volatilidade do preço do ativo e $\mathrm{dZ}=\varepsilon_{\mathrm{t}}(\mathrm{dt})^{1 / 2}$ é o incremento do processo padrão de Wiener com $\varepsilon_{\mathrm{t}} \sim \operatorname{NID}(0,1)$. Dado que $\mathrm{S}_{\mathrm{t}_{\mathrm{n}}}$ é uma variável estocástica a ser gerada por simulação de Monte Carlo, é conveniente utilizar um MGB discretizado, da seguinte forma:

$$
\mathrm{S}_{\mathrm{t}_{\mathrm{n}}+\Delta \mathrm{t}}=\mathrm{S}_{\mathrm{t}_{\mathrm{n}}} \exp \left[\left(\alpha-\sigma^{2} / 2\right) \Delta \mathrm{t}+\sigma(\Delta \mathrm{t})^{1 / 2} \varepsilon_{\mathrm{t}_{\mathrm{n}}+\Delta \mathrm{t}}\right]
$$

Onde $\Delta t$ é o intervalo de tempo entre $t_{n}$ e $t_{n+1}$. Desta maneira, é possível discretizar a equação (1) sob a mesma medida de probabilidade, resultando na seguinte equação:

$$
\mathrm{P}_{\mathrm{t}_{\mathrm{n}}}\left(\mathrm{S}_{\mathrm{t}_{\mathrm{n}}}, \mathrm{K}\right)=\frac{1}{\mathrm{M}} \sum_{\mathrm{a}=1}^{\mathrm{A}} \mathrm{e}^{-\left(\tau_{\mathrm{a}}^{*}-t_{\mathrm{n}}\right) \mathrm{r}}\left(\mathrm{K}-\mathrm{S}_{\tau_{\mathrm{a}}^{*}}\right)
$$


Onde A é o número total de caminhos Brownianos, dentre os $\mathrm{M}$ simulados a partir de um instante de tempo $t_{n}$, nos quais num instante de tempo (o primeiro) $t_{n+i}$ tem-se $S_{t_{n+i}} \leq S_{t_{n+i}} *$ (podem existir caminhos que em nenhum $t_{n+i}$ aconteça tal situação). Por conseqüencia existem " $\mathrm{A}$ " períodos $\tau^{*}$, identificados por $\tau^{*}{ }_{\mathrm{a}}(\mathrm{a}=1, \ldots, \mathrm{A})$ correspondentes a um determinado $\mathrm{S}_{\tau^{*}{ }_{\mathrm{a}}}$ em que a mencionada restrição é satisfeita.

\subsection{Descrição do Algoritmo de Ibáñez \& Zapatero}

\subsubsection{Determinar a fronteira de exercício ótima um período antes da maturidade}

No instante de tempo $\mathrm{t}_{\mathrm{N}}=\mathrm{T}$, o preço crítico de exercício $\mathrm{S}_{\mathrm{t}_{\mathrm{N}}}$ * é igual ao preço de exercício da opção, representado pela letra K. Por definição, o preço da opção de compra é a diferença entre o preço do ativo $S_{t_{i}}$ e o preço de exercício $K$ (ou seja $S_{t_{i}}-K$ ). Para a opção de venda temos $\mathrm{K}-\mathrm{S}_{\mathrm{t}_{\mathrm{i}}}$. Se no tempo $\mathrm{t}_{\mathrm{N}}=\mathrm{T}$ (último período para decidir o exercício) $\mathrm{S}_{\mathrm{t}_{\mathrm{i}}}$ é igual a $\mathrm{K}$, o valor da opção seria zero, portanto, exercer ou não a opção forneceria o mesmo resultado (zero para essa data). Assim, o preço crítico de exercício $\mathrm{S}_{\mathrm{t}_{\mathrm{N}}} *$ é igual a $\mathrm{K}$.

Portanto, o trabalho concentra-se em calcular recursivamente os outros pontos da curva de gatilho. Inicia-se então pelo preço crítico de exercício no período $t_{\mathrm{N}-1}$.

Passo 1: Deseja-se achar o ponto $\mathrm{S}_{\mathrm{t}_{\mathrm{N}-1}}$ *. Começa-se com um ponto inicial $\mathrm{S}_{\mathrm{t}_{\mathrm{N}-1}}{ }^{\text {(1) }}$ escolhido arbitrariamente. Normalmente toma-se este valor igual ao preço de exercício $\mathrm{K}$.

Passo 2: Depois, calcula-se o preço da opção: $\mathrm{P}_{\mathrm{t}_{\mathrm{N}-1}}\left(\mathrm{~S}_{\mathrm{t}_{\mathrm{N}-1}}^{(1)}, \mathrm{K}\right)$ em $\mathrm{t}_{\mathrm{N}-1}$, aplicando para isso a simulação de Monte Carlo conforme a equação (3). Por outro lado, e só no instante $\mathrm{t}_{\mathrm{N}-1}$, seria também possível empregar a conhecida fórmula de Black \& Scholes (1973), visto que entre $\mathrm{t}_{\mathrm{N}-1}$ e $\mathrm{t}_{\mathrm{N}}$ existe um só período. Assim, tomando como valor inicial do ativo $\mathrm{S}_{\mathrm{t}_{\mathrm{N}-1}}{ }^{(1)}$ e o preço de exercício $\mathrm{K}$ em $\mathrm{t}_{\mathrm{N}}$, calcula-se o preço da opção de venda com a exatidão que fornece esta fórmula, sem ser necessário (neste particular caso) realizar as simulações.

Passo 3: Para encontrar um novo preço $\mathrm{S}_{\mathrm{t}_{\mathrm{N}-1}}{ }^{(2)}$ que se aproxime mais do $\mathrm{S}_{\mathrm{t}_{\mathrm{N}-1}}$, é necessário encontrar uma regra eficiente para ir de um ponto de aproximação a outro. Uma forma bastante rápida para convergir ao preço crítico de exercício é utilizar o método de aproximações sucessivas de Newton. A convexidade da função preço da opção garante a convergência até o ponto fixado $\mathrm{S}_{\mathrm{t}_{\mathrm{N}-1}}{ }^{*}$. Assim:

- Para $\mathrm{s}=1,2,3, . .{ }^{*}$, usa-se a aproximação:

$$
\mathrm{P}_{\mathrm{t}_{\mathrm{N}-1}}\left(\mathrm{~S}_{\mathrm{t}_{\mathrm{N}-1}}^{(\mathrm{s})}, \mathrm{K}\right)+\mathrm{P}_{\mathrm{S}}\left(\mathrm{S}_{\mathrm{t}_{\mathrm{N}-1}}^{(\mathrm{s})}\right) \cdot\left(\mathrm{S}_{\mathrm{t}_{\mathrm{N}-1}}^{(\mathrm{s}+1)}-\mathrm{S}_{\mathrm{t}_{\mathrm{N}-1}}^{(\mathrm{s})}\right)=\mathrm{I}\left(\mathrm{S}_{\mathrm{t}_{\mathrm{N}-1}}^{(\mathrm{s}+1)}, \mathrm{K}\right)
$$

onde $\mathrm{P}_{\mathrm{S}}\left(\mathrm{S}_{\mathrm{t}_{\mathrm{N}-1}}^{(\mathrm{s})}\right)=\frac{\partial \mathrm{P}\left(\mathrm{S}_{\mathrm{t}_{\mathrm{N}-1}}^{(\mathrm{s})}, \mathrm{K}\right)}{\partial \mathrm{S}}$. Reorganizando a equação (4) encontra-se o valor $\mathrm{S}_{\mathrm{t}_{\mathrm{N}-1}}{ }^{(\mathrm{s}+1)}$ :

$$
\mathrm{S}_{\mathrm{t}_{\mathrm{N}-1}}^{(\mathrm{s}+1)}=\left(\mathrm{K}-\mathrm{P}_{\mathrm{t}_{\mathrm{N}-1}}\left(\mathrm{~S}_{\mathrm{t}_{\mathrm{N}-1}}^{(\mathrm{s})}, \mathrm{K}\right)+\mathrm{P}_{\mathrm{S}}\left(\mathrm{S}_{\mathrm{t}_{\mathrm{N}-1}}^{(\mathrm{s})}\right) \mathrm{S}_{\mathrm{t}_{\mathrm{N}-1}}^{(\mathrm{s})}\right) \div\left(1+\mathrm{P}_{\mathrm{S}}\left(\mathrm{S}_{\mathrm{t}_{\mathrm{N}-1}}^{(\mathrm{s})}\right)\right)
$$

Para resolver a equação (5) deve-se calcular antes $\mathrm{P}_{\mathrm{S}}\left(\mathrm{S}_{\mathrm{t}_{\mathrm{N}-1}}^{(\mathrm{s})}\right)$, que é a principal dificuldade na aplicação do método de Newton.

Pesquisa Operacional, v.28, n.3, p.473-490, Setembro a Dezembro de 2008 
O cálculo analítico de $\mathrm{P}_{\mathrm{S}}\left(\mathrm{S}_{\mathrm{t}_{\mathrm{N}-1}}^{(\mathrm{s})}\right)$ para uma opção de venda americana, a cada iteração 's', não é possível (só em opções européias existe expressão analítica para $\mathrm{P}_{\mathrm{S}}\left(\mathrm{S}_{\mathrm{t}_{\mathrm{N}-1}}^{(\mathrm{s})}\right)$ ). É um fato amplamente conhecido na literatura de Finanças que à medida que $\mathrm{S}_{\mathrm{t}_{\mathrm{n}}}(\mathrm{n} \in\{\mathrm{N}-1, \mathrm{~N}-2, \ldots, 1\})$ se aproxima mais do preço crítico de exercício $\mathrm{S}_{\mathrm{t}_{\mathrm{n}}}{ }^{*}$, a derivada do preço da opção de venda com respeito ao preço do ativo subjacente vai se aproximando do -1. Por esta razão, Ibáñez \& Zapatero (2004) sugerem iniciar as iterações com um valor $P_{S}\left(S_{t_{N-1}}^{(1)}\right)=-0.60$, e gradativamente realizar incrementos (negativos) a cada iteração até um máximo de $\mathrm{P}_{\mathrm{S}}\left(\mathrm{S}_{\mathrm{t}_{\mathrm{N}-1}}^{(*)}\right)=-0.90$.

Passo 4: Achado $\mathrm{S}_{\mathrm{t}_{\mathrm{N}-1}}^{(2)}$ calcula-se o preço da opção $\mathrm{P}_{\mathrm{t}_{\mathrm{N}-1}}\left(\mathrm{~S}_{\mathrm{t}_{\mathrm{N}-1}}^{(2)}, \mathrm{K}\right)$, aplicando a equação (3), ou a equação de Black \& Scholes (lembrado que esta só serve no período $t_{\mathrm{N}-1}$ ). Após, calcula-se uma nova aproximação $\mathrm{S}_{\mathrm{t}_{\mathrm{N}-1}}^{(3)}$ usando a equação (5).

Passo 5: Repete-se o procedimento 's' vezes até convergir ao valor $\mathrm{S}_{\mathrm{t}_{\mathrm{N}-1}}^{(*)}=\mathrm{S}_{\mathrm{t}_{\mathrm{N}-1}}^{(\mathrm{s})}$, sendo que $\left|\mathrm{S}_{\mathrm{t}_{\mathrm{N}-1}}^{(\mathrm{s})}-\mathrm{S}_{\mathrm{t}_{\mathrm{N}-1}}^{(\mathrm{s}-1)}\right|<\xi$, para algum número $\xi$ muito pequeno.

A convergência para o ponto fixado $\mathrm{S}_{\mathrm{t}_{\mathrm{N}-1}}^{(*)}$ se realiza de maneira monotônica, isto é, para uma opção de venda tem-se que: $\mathrm{S}_{\mathrm{t}_{\mathrm{N}-1}}^{(1)}>\mathrm{S}_{\mathrm{t}_{\mathrm{N}-1}}^{(2)}>\ldots>\mathrm{S}_{\mathrm{t}_{\mathrm{N}-1}}^{(*)}$. As iterações finalizam quando se encontra $\mathrm{S}_{\mathrm{t}_{\mathrm{N}-1}}^{(\mathrm{s}-1)}-\mathrm{S}_{\mathrm{t}_{\mathrm{N}-1}}^{(\mathrm{s})}<\xi$ ou quando exista uma mudança no sinal da convergência: $\mathrm{S}_{\mathrm{t}_{\mathrm{N}-1}}^{(\mathrm{s})}>\mathrm{S}_{\mathrm{t}_{\mathrm{N}-1}}^{(\mathrm{s}-1)}$. Em qualquer dos dois casos estima-se o ponto médio das duas últimas iterações: $\hat{S}_{t_{\mathrm{N}-1}}^{(*)}=\left(\mathrm{S}_{\mathrm{t}_{\mathrm{N}-1}}^{(\mathrm{s})}+\mathrm{S}_{\mathrm{t}_{\mathrm{N}-1}}^{(\mathrm{s}-1)}\right) / 2$, sendo esta a estimativa do valor de $\mathrm{S}_{\mathrm{t}_{\mathrm{N}-1}}^{(*)}$.

\subsubsection{Determinar a fronteira de exercício ótima nos períodos $\mathrm{t}_{\mathrm{N}-2}, \mathrm{t}_{\mathrm{N}-1}, \ldots, \mathrm{t}_{0}$}

Seguidamente, repete-se o mesmo procedimento para os pontos $t_{\mathrm{N}-2}, t_{\mathrm{N}-1}, \ldots, t_{0}$ (de maneira recursiva). A cada ponto, sugere-se reiniciar o algoritmo tomando como preço inicial do ativo $S_{t_{n}}^{(1)}=S_{t_{n+1}}^{(*)}$ (o ponto fixado do período à frente, calculado previamente).

Assim, no final encontra-se um conjunto de pontos $\mathrm{S}^{(*)}=\left\{\mathrm{S}_{\mathrm{t}_{0}}^{(*)}, \mathrm{S}_{\mathrm{t}_{1}}^{(*)}, \ldots, \mathrm{S}_{\mathrm{t}_{\mathrm{N}-1}}^{(*)}, \mathrm{S}_{\mathrm{t}_{\mathrm{N}}=\mathrm{T}}^{(*)}\right\}$, que formam a fronteira de exercício ótima ou curva de gatilho: $S_{t_{n}}^{(*)}=\hat{F}_{t_{n}}\left(t_{n}\right)$. Uma maneira eficiente de achar a função $\hat{\mathrm{F}}_{\mathrm{t}_{\mathrm{n}}}$ é fazendo uma regressão quadrática ou cúbica do conjunto $\mathrm{S}^{(*)}$ com os tempos $\left\{\mathrm{t}_{0}, \mathrm{t}_{1}, \ldots, \mathrm{T}\right\}$, embora resulte também aceitável fazer uma simples interpolação entre dois períodos discretos.

A utilidade da curva de gatilho está no fato que, no primeiro instante de tempo em que o valor do ativo subjacente "S" fique abaixo desta curva, dever-se-á optar pelo exercício, sendo o preço da opção de venda em algum instante $\mathrm{t}_{\mathrm{n}} \mathrm{o}$ valor intrínseco: $\mathrm{K}-\mathrm{S}_{\mathrm{t}_{\mathrm{n}}}$. 


\subsubsection{Calculando o preço da opção de venda}

Uma vez traçada a curva de gatilho, para calcular o preço da opção de venda, faz-se:

(i) Simular uma grande quantidade de caminhos (M) a partir do valor inicial do ativo $\mathrm{S}_{\mathrm{t}_{0}}=\mathrm{S}_{0}$. As simulações são feitas em intervalos de tempos discretos $\left\{\mathrm{t}_{1}, \mathrm{t}_{2}, \ldots, \mathrm{t}_{\mathrm{N}}=\mathrm{T}\right\}$.

(ii) Para cada caminho simulado, no primeiro instante de tempo $t_{n}$ em que o valor do ativo $\mathrm{S}_{t_{\mathrm{n}}}$ seja menor ou igual ao ponto fixado da curva de gatilho $\mathrm{S}_{t_{\mathrm{n}}}^{(*)}$, será exercida a opção, sendo o preço da opção de venda em $t_{n}$ o valor intrínseco: $\mathrm{K}-\mathrm{S}_{\mathrm{t}_{\mathrm{n}}}$. A seguir, desconta-se este preço com a taxa livre de risco: $\mathrm{P}_{\mathrm{m}}=\mathrm{e}^{-(\mathrm{tn}-\mathrm{t}) \mathrm{r}} \cdot\left(\mathrm{K}-\mathrm{S}_{\mathrm{t}_{\mathrm{n}}}\right)$, onde $\mathrm{P}_{\mathrm{m}}(\mathrm{m} \in\{1,2, \ldots, \mathrm{M}\})$ representa o preço da opção de venda em $\mathrm{t}_{0}$ para um caminho simulado dentre as ' $\mathrm{M}$ ' realizações. É provável que existam caminhos nos quais, em todo momento, os preços fiquem acima da curva de gatilho; para estes casos $\mathrm{P}_{\mathrm{m}}=0$, naturalmente.

(iii) O preço da opção de venda será a média aritmética de todos os $\mathrm{P}_{\mathrm{m}}$ 's:

$$
\mathrm{P}_{\mathrm{t}_{0}}\left(\mathrm{~S}_{0}, \mathrm{~K}\right)=1 / \mathrm{M} \sum_{\mathrm{m}=1}^{\mathrm{M}} \mathrm{P}_{\mathrm{m}}
$$

A Figura 1 apresenta um exemplo ilustrativo que calcula o preço de uma opção de venda com três caminhos simulados a partir de um preço inicial do ativo $\mathrm{S}_{0}=35$, em um horizonte de tempo dividido em seis períodos.

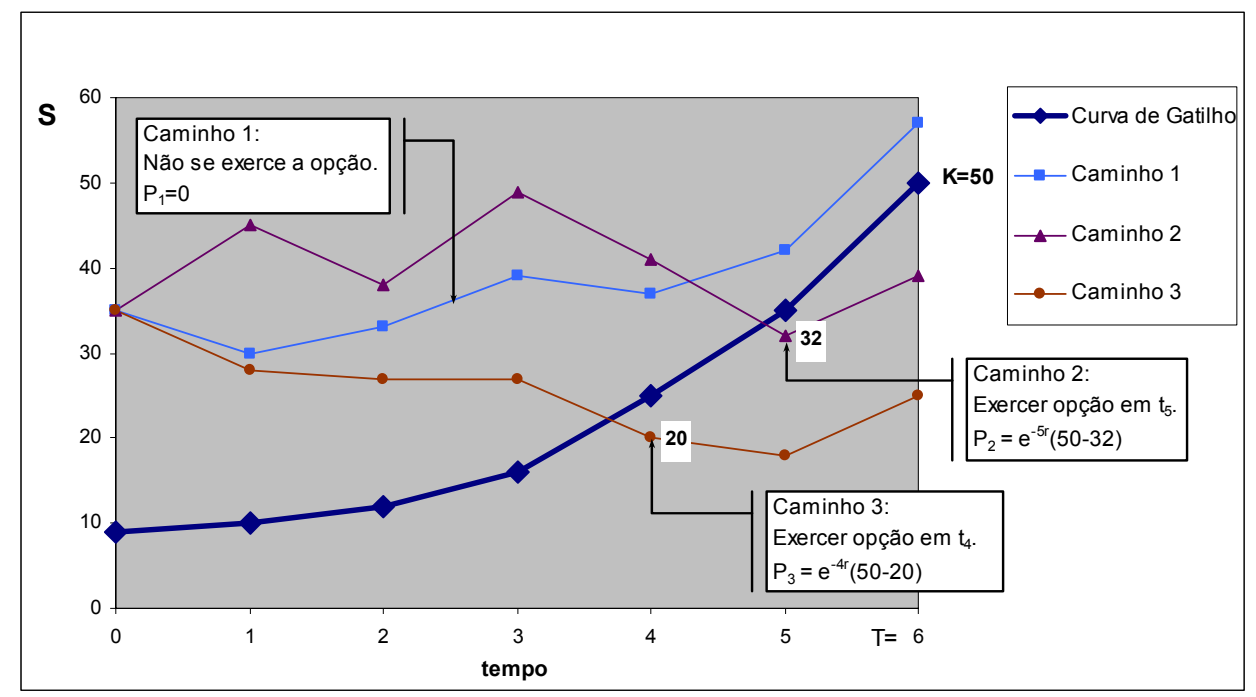

Figura 1 - Exemplo de Cálculo do Preço da Opção de Venda. Faz-se a média aritmética dos três $\mathrm{P}_{\mathrm{m}}$ 's. $\mathrm{P}_{0}\left(\mathrm{~S}_{0}=35, \mathrm{~K}=50\right)=\left(0+\mathrm{e}^{-5 \mathrm{r}}(50-32)+\mathrm{e}^{-4 \mathrm{r}}(50-20)\right) / 3$. 


\section{O Algoritmo de Ibáñez \& Zapatero Modificado}

Nesta seção apresentamos uma proposta de aprimoramento do algoritmo de Ibáñez \& Zapatero (2004). Posteriormente realizamos experimentos numéricos.

\subsection{Aprimoramento no cálculo da curva de gatilho}

A primeira melhoria que se pode efetuar no cálculo do ponto $\mathrm{S}_{\mathrm{t}_{\mathrm{N}-1}}^{(*)}$ é empregar a fórmula de Black \& Scholes (1973) para calcular o preço exato da opção de venda nos passos 2 e 4 do algoritmo, dado que existe um único período até a maturidade da opção. Ibáñez \& Zapatero (2004) também fazem esta sugestão. A novidade estaria no passo 3, onde deve ser estimado $\mathrm{P}_{\mathrm{S}}\left(\mathrm{S}_{\mathrm{t}_{\mathrm{N}-1}}^{(\mathrm{s})}\right)$. Quando existe só um período até a maturidade da opção, o valor da derivada do preço da opção com relação ao preço do ativo subjacente é calculado por uma expressão analítica fechada proveniente da fórmula de Black \& Scholes (1973) dada por:

$$
\mathrm{P}_{\mathrm{S}}\left(\mathrm{S}_{\mathrm{t}_{\mathrm{N}-1}}^{(\mathrm{s})}\right)=\mathrm{e}^{-\mathrm{q}\left(\mathrm{T}-\mathrm{t}_{\mathrm{N}-1}\right)}\left[\mathrm{N}\left(\mathrm{d}_{1}\right)-1\right]
$$

onde:

$$
\begin{aligned}
& \mathrm{d}_{1}=\left[\ln \left(\frac{\mathrm{S}_{\mathrm{t}_{\mathrm{N}-1}}^{(\mathrm{s}}}{\mathrm{K}}\right)+\left(\mathrm{r}-\mathrm{q}+\frac{1}{2} \sigma^{2}\right)\left(\mathrm{T}-\mathrm{t}_{\mathrm{N}-1}\right)\right] \frac{1}{\sigma \sqrt{\mathrm{T}-\mathrm{t}_{\mathrm{N}-1}}} \\
& \mathrm{~N}(.)=\text { função distribuição normal padrão acumulada. } \\
& \mathrm{q}=\text { taxa de dividendos. } \\
& \mathrm{r}=\text { taxa de desconto livre de risco. } \\
& \mathrm{K}=\text { preço de exercício. } \\
& \sigma=\text { volatilidade do preço do ativo subjacente. }
\end{aligned}
$$

Portanto, no passo 3 do algoritmo, referente ao período $\mathrm{t}_{\mathrm{N}-1}$ é conveniente usar a expressão analítica exata de $\mathrm{P}_{\mathrm{S}}\left(\mathrm{S}_{\mathrm{t}_{\mathrm{N}-1}}^{(\mathrm{s})}\right)$. Isto permitirá uma melhor convergência para o ponto $\mathrm{S}_{\mathrm{t}_{\mathrm{N}-1}}^{(*)}$.

Ibáñez \& Zapatero (2004) sugerem aplicar o algoritmo em duas etapas para obter um melhor cálculo da curva de gatilho nos períodos $\mathrm{t}_{\mathrm{N}-1}, \mathrm{t}_{\mathrm{N}-2}, \ldots, \mathrm{t}_{0}$. Seguindo esta sugestão, no algoritmo de Ibáñez \& Zapatero modificado também se aplicam as duas etapas nos mesmos períodos com exceção do ponto fixado no período $t_{\mathrm{N}-1}$, que já foi calculado empregando valores analíticos exatos do preço da opção de venda e da sua derivada. A seguir se explica em que consiste cada etapa.

\section{(i) Primeira etapa}

Determinar uma curva de gatilho simulando uma quantidade não muito grande de caminhos Brownianos aleatórios, por exemplo, 5000, a cada vez que se utilize a equação (3) nos passos 2 e 4. Para efeitos de redução de variância, as 5000 simulações devem estar constituídas por 2500 simulações mais seus respectivos valores antitéticos.

Variável antitética é uma das principais e mais simples técnicas para reduzir variância. Consiste em gerar uma variável estocástica negativamente correlacionada à variável de estado do ativo objeto. Assim, cada trajetória deve ser associada a um par de seqüências, isto é, duas trajetórias negativamente correlacionadas. Para maior informação sugere-se consultar Frota (2003). 
Melhoria proposta: No passo 3 do algoritmo, Ibáñez \& Zapatero (2004) não fornecem maiores detalhes de como fazer os acréscimos de $\mathrm{P}_{S}\left(\mathrm{~S}_{t_{n}}^{(s)}\right)$ a cada iteração. Eles sugerem começar com um valor $\mathrm{P}_{\mathrm{S}}\left(\mathrm{S}_{\mathrm{t}_{\mathrm{N}-1}}^{(1)}\right)=-0.60$, e depois fazer incrementos (negativos) a cada iteração até um valor máximo de $\mathrm{P}_{\mathrm{S}}\left(\mathrm{S}_{\mathrm{t}_{\mathrm{N}-1}}^{(*)}\right)=-0.90$. Mas, que valores devem ter tais incrementos?

A curva de gatilho a ser calculada nesta etapa é uma primeira tentativa de aproximar os preços críticos $\left\{\mathrm{S}_{\mathrm{t}_{0}}^{(*)}, \mathrm{S}_{\mathrm{t}_{1}}^{(*)}, \ldots, \mathrm{S}_{\mathrm{t}_{\mathrm{N}-3}}^{(*)}, \mathrm{S}_{\mathrm{t}_{\mathrm{N}-2}}^{(*)}\right\}$, assim, observou-se através de diversos experimentos numéricos, que uma maneira rápida de achar a convergência é realizando acréscimos de $-0,05(-\Delta=-0,05)$ começando com um valor de $P_{S}\left(S_{t_{n}}^{(1)}\right)=-0,60$. De acordo com as experimentações realizadas, na terceira ou quarta iteração já encontra-se o preço crítico de exercício, com um erro $\xi=0,01$ (erro de convergência descrito no passo 5 do algoritmo, que é do mesmo valor considerado por Ibáñez \& Zapatero (2004) nos testes que realizaram).

\section{(ii) Segunda etapa}

Os preços críticos de exercício achados na primeira etapa, servirão de ponto de partida $S_{t_{n}}^{(1)}$ a cada $t_{n}(n=\{0,1, \ldots, N-2\})$ em um novo cálculo da curva de gatilho. Assim, $S_{t_{n}}^{(1)}$ da etapa 2 é igual a $\mathrm{S}_{\mathrm{t}_{\mathrm{n}}}^{(*)}$ da etapa 1. Estes pontos iniciais estão muito mais próximos do verdadeiro valor $\mathrm{S}_{t_{n}}^{(*)}$, e conseqüentemente $\mathrm{P}_{\mathrm{S}}\left(\mathrm{S}_{t_{n}}^{(s)}\right)$ se aproxima mais de -1. Portanto, ao aplicar novamente o algoritmo espera-se obter uma melhor aproximação da curva de gatilho.

Para melhorar ainda mais a precisão, foram simulados 100000 caminhos Brownianos aleatórios (50000 mais seus respectivos valores antitéticos) a cada vez que se utilize a equação (3) nos passos 2 e 4.

Melhoria proposta: Nesta etapa, Ibáñez \& Zapatero (2004) não falam como fazer os acréscimos em $\mathrm{P}_{\mathrm{S}}\left(\mathrm{S}_{\mathrm{t}_{\mathrm{n}}}^{(\mathrm{s})}\right)$. Então, visto que os pontos iniciais encontram-se muito próximos dos preços críticos de exercício, diversos testes práticos efetuados indicam que, começando as iterações com um $\mathrm{P}_{\mathrm{S}}\left(\mathrm{S}_{t_{\mathrm{n}}}^{(1)}\right)=-0,85$ e fazendo posteriormente incrementos $(-\Delta)$ pequenos de $-0,01$, consegue-se uma boa aproximação. No máximo em 10 iterações encontrar-se-á o novo preço crítico de exercício, considerando um erro $\xi=0,01$.

\subsection{Experimentos utilizando o algoritmo de Ibáñez \& Zapatero modificado}

Ibáñez \& Zapatero (2004) fizeram testes numéricos com os mesmos parâmetros dos realizados por Huang, Subrahmanyam \& Yu (1996), que utilizaram o método binomial com 10000 passos, e cujos resultados são usados como benchmark. Para opções americanas com uma só variável estocástica, cujo ativo subjacente segue um MGB (Movimento Geométrico Browniano), o método binomial é uma maneira simples de avaliar estas opções, sendo seus resultados mais precisos quanto maior for a quantidade de divisões (passos) da árvore. Os exemplos numéricos a serem apresentados são opções de venda simples que o método binomial avalia bem, por isso é tomado como benchmark. A vantagem do uso de métodos de 
simulação de Monte Carlo para o cálculo de opções americanas é a sua maior flexibilidade, podendo simular simultaneamente diversas variáveis, seguindo variados processos estocásticos, o qual não é possível modelar pelo método binomial. Mas para efeitos de avaliar o grau de precisão de uma metodologia por simulação, simplificam-se os testes de tal maneira que se consigam modelar pelo método binomial, e os seus resultados servirão para poder comparar com os resultados que a metodologia por simulação oferece nos mesmos testes.

Na Tabela 1 são exibidos doze testes numéricos nos quais é calculado o preço da opção de venda americana. Os parâmetros comuns são os seguintes: $r=0,0488$ anual (taxa livre de risco) $\delta=0$ (taxa de dividendos). Aplicam-se as duas etapas (como foram descritas) na determinação da curva de gatilho. Uma vez obtida a curva, para calcular o preço da opção de venda simulam-se 100000 caminhos Brownianos (50000 com seus valores antitéticos) a partir do preço inicial do ativo $\mathrm{S}_{0}=40$. Simulam-se estes caminhos 50 vezes (sob a mesma curva de gatilho calculada previamente), e o preço da opção de venda provém da média aritmética do preço da opção obtido a cada vez em que se realizaram as 100000 simulações de caminhos.

Tabela 1 - Resultados do algoritmo de Ibáñez \& Zapatero modificado. $\left(\mathrm{S}_{0}=40 ; \mathrm{r}=0,0488 ; \delta=0\right) * *$

\begin{tabular}{|c|c|c|c|c|c|c|c|c|c|}
\hline \multirow[b]{2}{*}{ K } & \multirow[b]{2}{*}{$\sigma$} & \multirow[b]{2}{*}{$\mathrm{T}$ (anos) } & \multirow[b]{2}{*}{$\begin{array}{l}\text { Opção de } \\
\text { Venda } \\
\text { Verdadeira* }\end{array}$} & \multicolumn{3}{|c|}{5 datas de exercício } & \multicolumn{3}{|c|}{25 datas de exercício } \\
\hline & & & & $\begin{array}{l}\text { Preço } \\
\text { Opção de } \\
\text { Venda }\end{array}$ & $\begin{array}{l}\text { Desvio } \\
\text { Padrão }\end{array}$ & $\begin{array}{c}\% \\
\text { diferença }\end{array}$ & $\begin{array}{l}\text { Preço } \\
\text { Opção de } \\
\text { Venda }\end{array}$ & $\begin{array}{l}\text { Desvio } \\
\text { Padrão }\end{array}$ & $\begin{array}{c}\% \\
\text { diferença }\end{array}$ \\
\hline 35 & 0,2 & 0,0833 & 0,0062 & 0,0062 & 0,0003 & $0,00 \%$ & 0,0062 & 0,0002 & $0,00 \%$ \\
\hline 35 & 0,2 & 0,5833 & 0,4328 & 0,4263 & 0,0035 & $1,50 \%$ & 0,4315 & 0,0038 & $0,30 \%$ \\
\hline 40 & 0,2 & 0,0833 & 0,8522 & 0,8487 & 0,0023 & $0,41 \%$ & 0,8512 & 0,0019 & $0,12 \%$ \\
\hline 40 & 0,2 & 0,5833 & 1,9904 & 1,9654 & 0,0046 & $1,26 \%$ & 1,9851 & 0,0050 & $0,27 \%$ \\
\hline 45 & 0,2 & 0,0833 & 5,0000 & 4,9659 & 0,0011 & $0,68 \%$ & 4,9927 & 0,0002 & $0,15 \%$ \\
\hline 45 & 0,2 & 0,5833 & 5,2670 & 5,2076 & 0,0053 & $1,13 \%$ & 5,2562 & 0,0058 & $0,21 \%$ \\
\hline 35 & 0,4 & 0,0833 & 0,2466 & 0,2465 & 0,0027 & $0,04 \%$ & 0,2467 & 0,0026 & $0,04 \%$ \\
\hline 35 & 0,4 & 0,5833 & 2,1549 & 2,1408 & 0,0075 & $0,65 \%$ & 2,1528 & 0,0089 & $0,10 \%$ \\
\hline 40 & 0,4 & 0,0833 & 1,7681 & 1,7654 & 0,0040 & $0,15 \%$ & 1,7676 & 0,0046 & $0,03 \%$ \\
\hline 40 & 0,4 & 0,5833 & 4,3526 & 4,3243 & 0,0093 & $0,65 \%$ & 4,3477 & 0,0084 & $0,11 \%$ \\
\hline 45 & 0,4 & 0,0833 & 5,2868 & 5,2791 & 0,0054 & $0,15 \%$ & 5,2853 & 0,0045 & $0,03 \%$ \\
\hline 45 & 0,4 & 0,5833 & 7,3830 & 7,3406 & 0,0090 & $0,57 \%$ & 7,3748 & 0,0094 & $0,11 \%$ \\
\hline \multirow{2}{*}{ (5) } & & & & & MAPE & $0,5997 \%$ & & MAPE & $0,1211 \%$ \\
\hline & & & & & RMSE & $2,6196 \%$ & & RMSE & $0,4991 \%$ \\
\hline
\end{tabular}

* Os preços verdadeiros da opção de venda americana em cada teste numérico (12 em total) são os obtidos por Huang, Subrahmanyam \& Yu (1996) usando um modelo binomial com 10000 passos. Estes resultados servem como benchmark para o cálculo das medidas de erro: MAPE e RMSE.

** $\mathrm{S}_{0}$ é o valor inicial do ativo, $\mathrm{r}$ é a taxa livre de risco, e $\delta$ é a taxa de dividendos.

A seguir são apresentadas as explicações referentes à Tabela 1:

- O número de datas de exercício refere-se à quantidade de intervalos discretos em que o espaço de tempo compreendido entre $\mathrm{t}_{0}=0$ e $\mathrm{t}_{\mathrm{N}}=\mathrm{T}$ foi subdividido. Foram consideradas $5 \mathrm{e}$ 25 datas de exercício para efeitos de comparar com os resultados obtidos por Ibáñez \& Zapatero (2004), os quais fizeram os testes somente com essas datas. 
- A título de exemplificação, o primeiro teste numérico tem como parâmetros $\mathrm{K}=35, \sigma=0,2$, $\mathrm{T}=1$ mês $(0,0833$ anos). O preço médio da opção de venda calculado pelo algoritmo de Ibáñez \& Zapatero modificado (com 5 datas de exercício) resultou igual ao do benchmark, com um desvio padrão de 0,0003 .

- A coluna "\% diferença" mede a porcentagem, em valor absoluto, em que o preço da opção de venda calculado por meio das simulações se distancia do valor verdadeiro. Sua fórmula é: |Preço Opção de Venda - Opção de Venda Verdadeira//(Opção de Venda Verdadeira).

- O MAPE é uma medida estatística do erro para um conjunto de testes. No total foram realizados 12 testes numéricos (um em cada linha da Tabela 1). Em cada teste a "\% de diferença" varia. Portanto, uma maneira de consolidar uma medida de erro para um conjunto de testes realizados sob certos parâmetros comuns e um determinado número de datas de exercício, é por meio desta medida. Numericamente, o MAPE é a média aritmética da coluna "\% diferença" (explicada no parágrafo anterior).

- O RMSE é outra medida estatística do erro de um conjunto de testes, que para o caso de 12 testes sua fórmula seria: RMSE $=\sqrt{\sum_{i=1}^{12}\left(\text { Preço Put }_{\mathrm{i}}-\text { Put Verdadeira }_{\mathrm{i}}\right)^{2} / 12} \cdot$ Em outras palavras, é a raiz quadrada do erro médio quadrático.

- Chame-se de "experimento" ao conjunto de testes numéricos realizados sob uma certa quantidade de datas de exercício. Por exemplo, a Tabela 1 exibe dois experimentos, os quais agrupam 12 diferentes testes numéricos. Em cada experimento o conjunto de testes numéricos são os mesmos, só varia o valor do parâmetro "número de datas de exercício".

- Quanto menor for a porcentagem nestas medidas de erro, isto indicará que os valores estipulados nas variáveis do experimento, estão permitindo obter uma melhor aproximação para os valores de referência ou benchmarks. Nos experimentos apresentados na Tabela 1 se pode distinguir que um maior número de datas de exercício (esta é a variável que muda de um experimento para outro) faz que os valores do MAPE e do RMSE sejam reduzidos.

\subsection{Resultados dos testes feitos em Ibáñez \& Zapatero}

Nesta seção se realiza uma comparação baseada nos resultados das medidas de erro entre os algoritmos de Ibáñez \& Zapatero (2004) e Ibáñez \& Zapatero modificado, sendo que em ambos os casos são considerados os testes numéricos da Tabela 1. Na Tabela 2, são exibidos tais resultados.

Tabela 2 - Medidas de erro: Ibáñez \& Zapatero (I\&Z) versus I\&Z Modificado.

\begin{tabular}{|c|c|c|c|c|}
\cline { 2 - 5 } \multicolumn{1}{c|}{} & \multicolumn{2}{c|}{5 datas de exercício } & 25 datas de exercício \\
\hline Algoritmo utilizado & MAPE & RMSE & MAPE & RMSE \\
\hline Ibáñez \& Zapatero $(I \& Z)^{*}$ & $1,0818 \%$ & $2,7295 \%$ & $0,6151 \%$ & $0,9567 \%$ \\
\hline I\&Z Modificado** & $0,5997 \%$ & $2,6196 \%$ & $0,1211 \%$ & $0,4991 \%$ \\
\hline
\end{tabular}
Fonte: * Elaboração própria a partir dos resultados obtidos por I\&Z.
$* *$ Resultados provenientes da Tabela 1.

Pesquisa Operacional, v.28, n.3, p.473-490, Setembro a Dezembro de 2008 
Observa-se na Tabela 2, que o algoritmo modificado I\&Z gera menores erros em ambas as datas de exercício. Desta maneira, verifica-se que as modificações realizadas no algoritmo de Ibáñez \& Zapatero (2004) foram apropriadas. Esta melhora na precisão dos resultados é devido ao aprimoramento realizado na determinação da curva de gatilho. Na estimação do ponto crítico de exercício do penúltimo período, por exemplo, ao invés de simular caminhos entre uma iteração e outra até atingir o preço crítico de exercício, emprega-se a fórmula de Black \& Scholes (1973), e, além disso, ao utilizar as aproximações sucessivas de Newton usou-se a fórmula analítica da derivada da opção. Por outro lado, na estimação dos outros pontos críticos de exercício, o fato de que em cada uma das duas etapas de aplicação do algoritmo ter sido utilizado diferentes valores inicias da derivada e dos acréscimos (passo 3 do algoritmo), contribuiu também para obter uma melhor aproximação.

\subsection{Análise de sensibilidade do algoritmo de I\&Z modificado}

\subsubsection{Número de datas de exercício}

Pelo exibido na Tabela 2, observa-se que o número de datas de exercício melhora a precisão dos resultados, obtendo menores valores dos erros.

Foram realizados experimentos adicionais alterando-se o valor desta variável. Os testes numéricos são sempre os exibidos na Tabela 1, mantendo os mesmos parâmetros para o cálculo da curva de gatilho e para o cálculo do preço da opção de venda.

Os resultados dessas experimentações são exibidos a seguir na Figura 2.

De acordo com a Figura 2, nota-se que existe uma significativa redução das medidas de erro à medida que o número de datas de exercício vai crescendo até um valor aproximado de 25 . A partir daí a redução das medidas de erro continua, mas já não é tão significativo o ganho obtido pelo acréscimo de maior quantidade de datas.

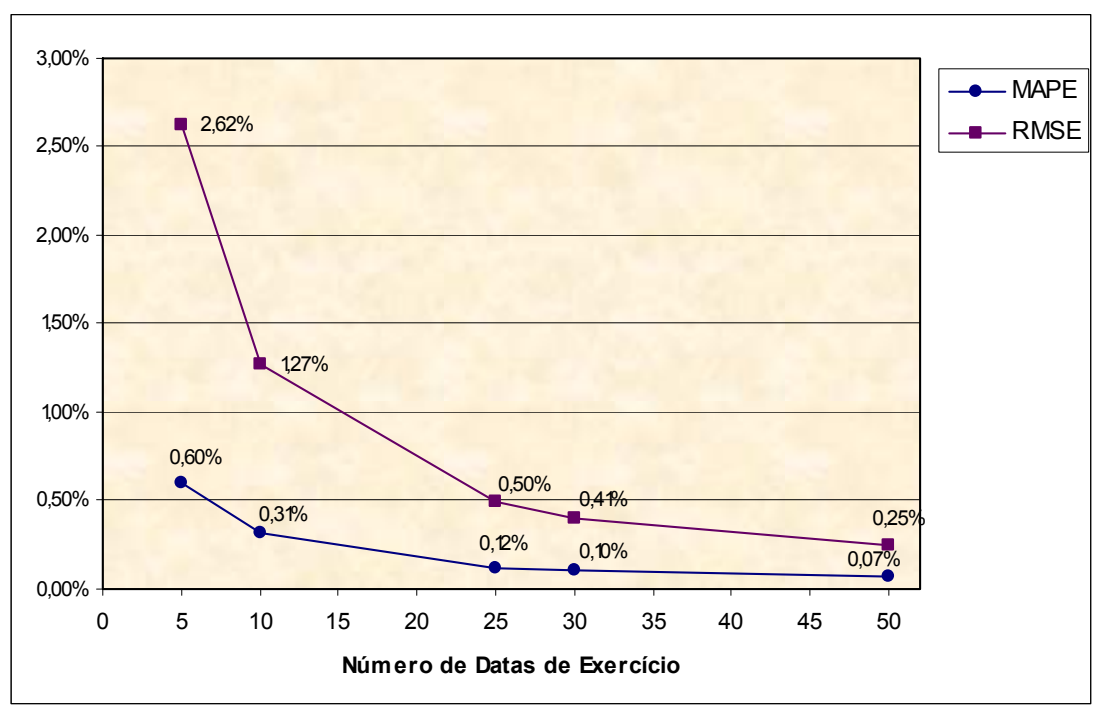

Figura 2 - Valor das medidas de erro em função do número de datas de exercício. 
O tempo de processamento computacional cresce à medida que se incrementa o número de datas de exercício. A Figura 3 exibe os tempos empregados em média para calcular o preço da opção de venda americana de um teste numérico, em função do número de datas de exercício.

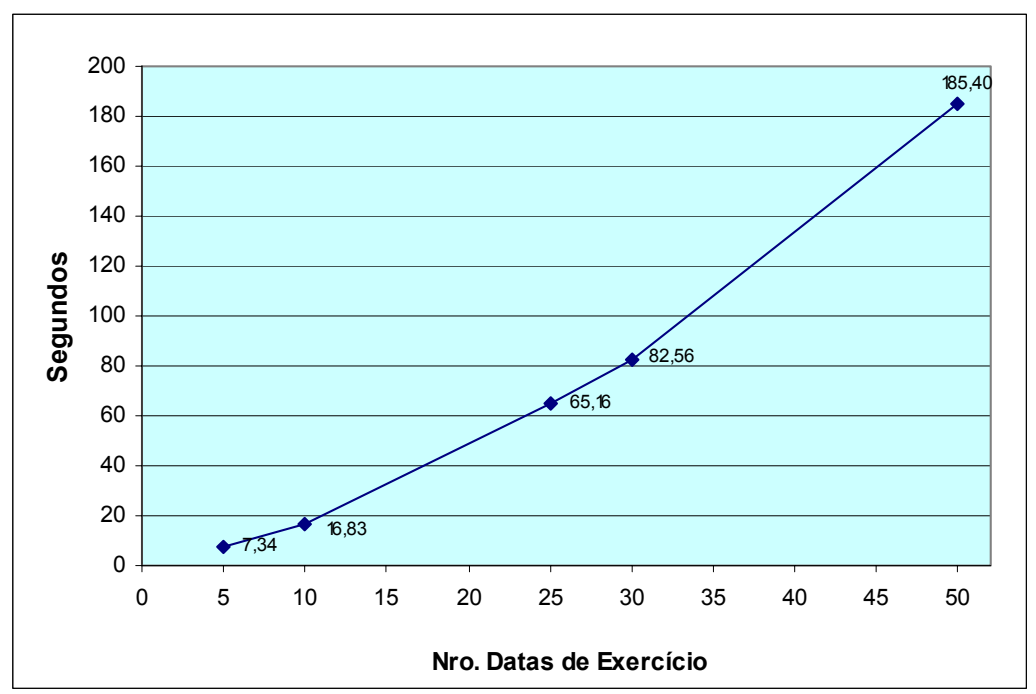

Figura 3 - Tempo computacional em função do número de datas de exercício.

Em relação ao tempo de processamento computacional nota-se um significativo aumento à medida que o número de datas de exercício é maior. Pelo exibido na Figura 3, o tempo que se leva em computar em média um teste numérico com 25 datas de exercício é de 65,16 segundos ( 1 minuto aprox.), mas ao se dobrar o número de datas de exercício (50 datas) o tempo de processamento sobe para 185,40 segundos (3 minutos aproximadamente). $\mathrm{O}$ ganho na melhora do erro de passar de 25 para 50 datas talvez não justifique o custo computacional.

Cabe anotar que o programa que computou os diferentes testes numéricos realizados neste trabalho foi desenvolvido em MatLab, rodado em um computador Pentium IV de 2,8 GHz e 480 MB de RAM.

\subsubsection{Outras análises de sensibilidade}

Foram realizados experimentos numéricos para analisar o comportamento de algumas variáveis, por exemplo, o número de simulações de caminhos Brownianos do preço do ativo subjacente, que permite calcular o preço da opção de venda americana uma vez que se tenha determinado a curva de gatilho. Dividindo o tempo até a maturidade em 25 datas de exercício, constatou-se que a partir de 10000 simulações consegue-se uma estabilidade nas medidas de erro MAPE e RMSE, e, em relação ao tempo de processamento computacional, existe uma diferença de poucos segundos entre o que se consome com 10000 e 100000 simulações. No entanto, optou-se por fazer os testes com 100000 simulações, pois Ibáñez \& Zapatero (2004) utilizaram essa quantidade de simulações. Desta forma, e para efeitos de comparação, manteve-se o mesmo valor do parâmetro. 
Adicionalmente realizou-se um experimento para analisar se a média de várias curvas de gatilho melhoraria a precisão dos resultados. Os resultados mostraram que o fato de calcular várias curvas de gatilho e com estas se obter uma curva média (tirando a média dos valores dos preços críticos de exercício em cada instante de tempo), aquilo não implicou em uma melhora nas medidas de erro. Conclui-se então que basta calcular uma única curva aplicando sempre as duas etapas descritas. A média de várias curvas de gatilho resultou ser desnecessária aumentando exponencialmente o tempo de processamento computacional.

\section{O Método dos Mínimos Quadrados de Monte Carlo (LSM)}

A metodologia dos Mínimos Quadrados de Monte Carlo, mais conhecida como LSM (Least Square Monte Carlo) desenvolvida por Longstaff \& Schwartz (2001), alcançou nos últimos anos uma grande aceitação, pela sua facilidade de cálculo e por fornecer resultados bem próximos do real. Utiliza também o critério da programação dinâmica para calcular o preço da opção, sem precisar determinar previamente a curva de gatilho. Sugere-se consultar Frota (2003), Araújo (2004) e Nascimento (2005) para uma explicação mais detalhada sobre $\mathrm{o}$ algoritmo.

\subsection{Testes numéricos com o algoritmo LSM}

Para testar a metodologia LSM consideraram-se novamente os testes numéricos mostrados na Tabela 1.

Como parâmetros, dividiu-se o período até a maturidade em 25 datas de exercício, além disso foram simulados 20000 caminhos Brownianos (10000 com seus valores antitéticos), sendo que ao aplicar o algoritmo LSM a um determinado teste numérico obteve-se um preço da opção de venda. Com 20000 simulações consegue-se obter uma boa convergência para o preço verdadeiro da opção de venda americana, como foi detectado através de uma análise de sensibilidade que será explicada na seção seguinte. Repetiu-se este procedimento 50 vezes, e o preço da opção de venda mostrado na Tabela 3, é a média aritmética dos cinqüenta preços obtidos a cada vez. O desvio padrão foi calculado daquela amostra. Na Tabela 3 são exibidos os resultados alcançados em todos os testes.

\subsection{Análises de sensibilidade do algoritmo LSM}

Uma primeira análise de sensibilidade tem como finalidade descobrir o comportamento das medidas de erro em função do número de caminhos simulados a partir de um preço inicial do ativo base $\left(\mathrm{S}_{0}\right)$. O algoritmo LSM dispensa o cálculo prévio da curva de gatilho, e, a partir daquelas simulações, aplicando um procedimento recursivo, consegue-se calcular o preço da opção americana. Foram realizadas experimentações com 25 datas de exercício e utilizando uma regressão polinomial linear de grau 2. Observou-se que partir de 20000 simulações que se consegue uma estabilidade nas medidas de erro. Daí o motivo pelo qual os testes numéricos da Tabela 3 foram realizados com esse número de simulações. 
Tabela 3 - Resultados aplicando o algoritmo LSM. $\left(\mathrm{S}_{0}=40 ; \mathrm{r}=0,0488 ; \delta=0 ; 25 \text { datas de exercício }\right)^{* *}$

\begin{tabular}{|c|c|c|c|c|c|c|}
\hline $\mathrm{K}$ & $\sigma$ & $\mathrm{T}$ (anos) & $\begin{array}{c}\text { Opção de } \\
\text { Venda } \\
\text { Verdadeira* }\end{array}$ & $\begin{array}{c}\text { Preço Opção } \\
\text { de Venda }\end{array}$ & $\begin{array}{c}\text { Desvio } \\
\text { Padrão }\end{array}$ & $\begin{array}{c}\% \\
\text { diferença }\end{array}$ \\
\hline 35 & 0,2 & 0,0833 & 0,0062 & 0,0061 & 0,0002 & $1,61 \%$ \\
\hline 35 & 0,2 & 0,5833 & 0,4328 & 0,4300 & 0,0032 & $0,65 \%$ \\
\hline 40 & 0,2 & 0,0833 & 0,8522 & 0,8499 & 0,0022 & $0,27 \%$ \\
\hline 40 & 0,2 & 0,5833 & 1,9904 & 1,9841 & 0,0040 & $0,32 \%$ \\
\hline 45 & 0,2 & 0,0833 & 5,0000 & 4,9927 & 0,0001 & $0,15 \%$ \\
\hline 45 & 0,2 & 0,5833 & 5,2670 & 5,2559 & 0,0063 & $0,21 \%$ \\
\hline 35 & 0,4 & 0,0833 & 0,2466 & 0,2457 & 0,0026 & $0,36 \%$ \\
\hline 35 & 0,4 & 0,5833 & 2,1549 & 2,1496 & 0,0091 & $0,25 \%$ \\
\hline 40 & 0,4 & 0,0833 & 1,7681 & 1,7651 & 0,0038 & $0,17 \%$ \\
\hline 40 & 0,4 & 0,5833 & 4,3526 & 4,3457 & 0,0086 & $0,16 \%$ \\
\hline 45 & 0,4 & 0,0833 & 5,2868 & 5,2781 & 0,0055 & $0,16 \%$ \\
\hline 45 & 0,4 & 0,5833 & 7,3830 & 7,3733 & 0,0079 & $0,13 \%$ \\
\hline & & & & MAPE & $\mathbf{0 , 3 6 9 8 \%}$ \\
\cline { 2 - 6 } & & & RMSE & $\mathbf{0 , 6 3 5 5 \%}$ \\
\hline
\end{tabular}

* Os preços verdadeiros da opção de venda americana em cada teste numérico (12 em total) são os obtidos por Huang, Subrahmanyam \& Yu (1996) usando um modelo binomial com 10000 passos. Estes resultados servem como benchmark para o cálculo das medidas de erro: MAPE e RMSE.

** $\mathrm{S}_{0}$ é o valor inicial do ativo, $\mathrm{r}$ é a taxa livre de risco, e $\delta$ é a taxa de dividendos.

Existe uma significativa diferença no tempo de processamento computacional entre 20000 e 100000 simulações; o primeiro caso requer em média 37 segundos para efetuar um teste numérico; o segundo caso, são necessários 193 segundos. Conseqüentemente, não é preciso trabalhar com um número elevado de simulações, não houve melhora nos resultados e há maior demanda de tempo.

Uma análise de sensibilidade adicional consistiu em examinar se o grau do polinômio de regressão influencia na precisão dos resultados. Foram feitos diversos experimentos para os doze testes numéricos (empregando 20000 simulações e 25 datas de exercício), sendo que em cada experimento alterou-se o grau do polinômio. Os resultados mostraram que o fato de acrescentar o valor de este parâmetro não melhora as medidas de erro, e só faz aumentar o tempo de processamento computacional. Portanto, utilizando um grau de polinômio igual a dois, é suficientemente aceitável para a realização dos experimentos.

\section{Análise dos Resultados e Comparações das Metodologias Testadas}

A Figura 4 apresenta um resumo das medidas de erro nos três algoritmos testados, sendo que o tempo até a maturidade foi dividido em 25 datas de exercício, o que mostrou ser uma quantidade razoável para obter boa precisão nos resultados. Observa-se que o algoritmo I\&Z modificado gera as menores medidas de erro em relação às outras metodologias abordadas. 
No que diz respeito ao tempo de processamento computacional (ver Figura 5) o algoritmo LSM demanda um tempo menor, isto porque não é necessário fazer um cálculo prévio da curva de gatilho, o qual economiza esforços computacionais. Esses tempos são referidos ao que se gasta em média para realizar um teste numérico com os parâmetros que originaram as medidas mostradas na Figura 4; isto é com 25 datas de exercício (nos três casos), 100000 simulações para os algoritmos I\&Z e I\&Z modificado, e 20000 simulações com polinômio de regressão de grau 2 no algoritmo LSM.

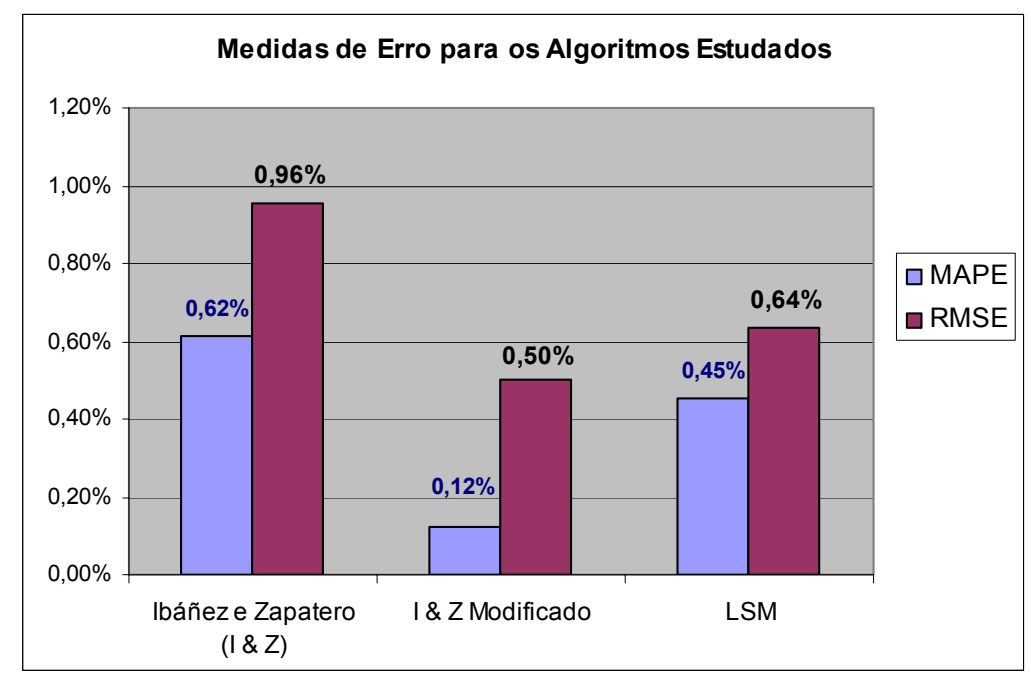

Figura 4 - Medidas de erro nos três algoritmos abordados.

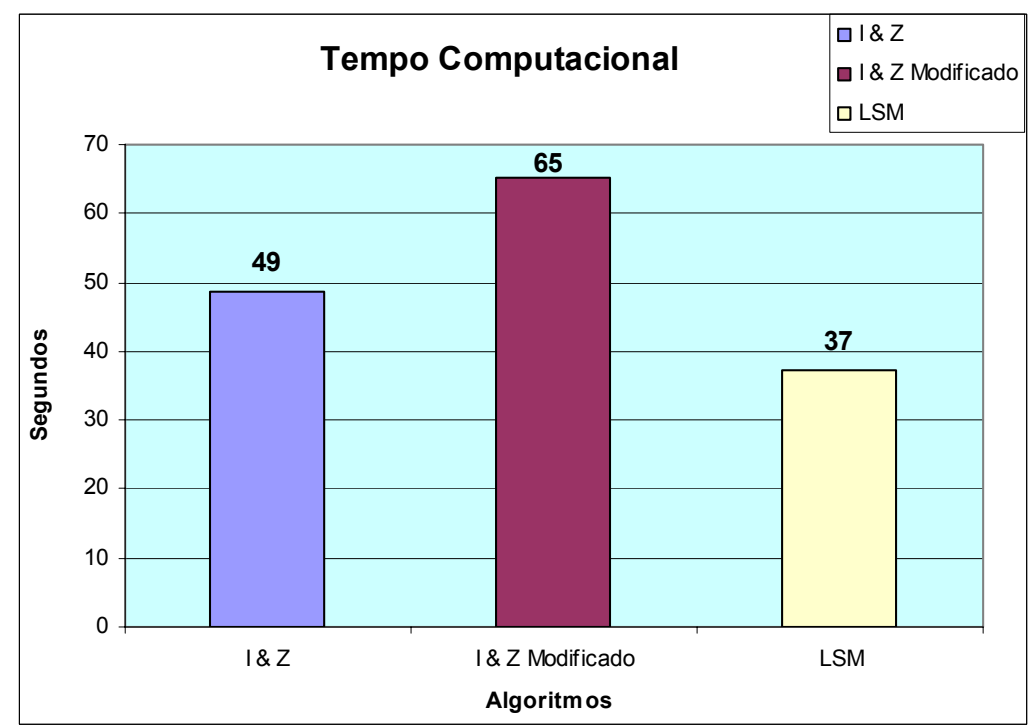

Figura 5 - Tempo de processamento computacional dos algoritmos. 


\section{Conclusões e Considerações Finais}

Neste trabalho foram avaliados os algoritmos de Ibáñez \& Zapatero (2004) e LSM de Longstaff \& Schwartz (2001). Simultaneamente foram propostas modificações no primeiro algoritmo de forma a obter maior precisão nos resultados do preço da opção de venda americana conforme foi mostrado na Figura 4.

A proposta feita para melhorar a convergência para os preços críticos de exercício, estabelecendo valores iniciais das derivadas do preço da opção e dos incrementos a cada iteração (passo 3 do algoritmo I\&Z), demonstrou ser bastante eficiente, permitindo melhorar os resultados obtidos por Ibáñez \& Zapatero (2004). Também mostrou-se que o algoritmo de Ibáñez $\&$ Zapatero modificado obteve erros um pouco menores que aqueles do algoritmo LSM.

Com respeito do tempo computacional, a metodologia LSM resulta ser mais vantajosa, mas o algoritmo modificado de Ibáñez \& Zapatero, sob determinados parâmetros, ofereceu resultados com a melhor aproximação para o benchmark nos testes realizados. O algoritmo LSM pode ser mais rápido e fornece resultados muito satisfatórios, porém não calcula a curva de gatilho (a sua determinação envolveria um tempo computacional muito elevado), que em algumas situações resulta ser mais importante do que conhecer o preço da opção. Portanto, dependendo dos objetivos que sejam perseguidos, uma ou outra metodologia poderá ser mais adequada. Cabe ressaltar que com o passar dos anos, o tempo de processamento computacional está sendo cada vez menos relevante.

O estudo feito no presente trabalho pode ser estendido para o caso de opções de compra americanas, situações em que existam duas ou mais variáveis estocásticas, aplicações em opções reais e na análise do tempo ótimo para investimentos, mencionando algumas das principais extensões que podem ser consideradas.

\section{Referências Bibliográficas}

(1) Araújo, R.O. (2004). Avaliação de Op̧̧ões Reais Através do Método dos Mínimos Quadrados de Monte Carlo. Dissertação de Mestrado, Departamento de Engenharia Industrial, PUC-Rio, Rio de Janeiro.

(2) Black, F. \& Scholes, M. (1973). The Pricing of Options and Corporate Liabilities. Journal of Political Economy, 81, 637-659.

(3) Boyle, P. \& Broadie, M. \& Glasserman, P. (1997). Monte Carlo Methods for Security Pricing. Journal of Economic Dynamics and Control, 21, 1267-1321.

(4) Broadie, M. \& Glasserman, P. (1997). Pricing American-Style Securities Using Simulation. Journal of Economic Dynamics and Control, 21, 1323-1352.

(5) Frota, A.E.F. (2003). Avaliação de Opções Americanas Tradicionais e Complexas. Dissertação de Mestrado, Departamento de Engenharia Industrial, PUC-Rio, Rio de Janeiro.

(6) Grant, D. \& Vora, G. \& Weeks, D.E. (1997). Path-Dependent Options: Extending the Monte Carlo Simulation Approach. Management Science, 43, 1589-1602.

(7) Huang, J.; Subrahmanyam, M.G. \& Yu, G.G. (1996). Pricing and Hedging American Options; A Recursive Integration Method. Review of Financial Studies, 9, 277-300.

Pesquisa Operacional, v.28, n.3, p.473-490, Setembro a Dezembro de 2008 
(8) Ibáñez, A. \& Zapatero, F. (2004). Monte Carlo Valuation of American Options Through Computation of the Optimal Exercise Frontier. Journal of Financial and Quantitative Analysis, 39, 253-275.

(9) Longstaff, F.A. \& Schwartz, E.S. (2001). Valuing American Options By Simulation: A Simple Least-Square Approach. Review of Financial Studies, 14, 113-147.

(10) Nascimento, A.F. (2005). Avaliação de Investimentos em Tecnologia da Informação: uma Perspectiva de Opções Reais. Dissertação de Mestrado, Departamento de Engenharia Industrial, PUC-Rio, Rio de Janeiro. 